\title{
Distance-Dependent Scaling of Calcium Transients Evoked by Backpropagating Spikes and Synaptic Activity in Dendrites of Hippocampal Interneurons
}

\author{
Balazs Rozsa, Tibor Zelles, E. Sylvester Vizi, and Balazs Lendvai \\ Institute of Experimental Medicine, Hungarian Academy of Sciences, H-1083 Budapest, Hungary
}

\begin{abstract}
Although interactions between backpropagating action potentials and synaptic stimulations have been extensively studied in pyramidal neurons, dendritic propagation and the summation of these signals in interneurons are not nearly as well known. In this study, twophoton imaging was used to explore the basic properties of dendritic calcium signaling in CA1 stratum radiatum interneurons. In contrast to hippocampal pyramidal neurons, the backpropagating action potential-evoked calcium transients in dendrites of interneurons underwent a distance-dependent increment. Although, in proximal dendrites, an increment could be attributed to a smaller dendrite diameter, distal dendrites did not show such dependence. Calcium responses in interneurons had a smaller amplitude, slower rise time, and decay than in pyramidal neurons. To explore the factors underlying the difference, we compared the calcium-binding capacity in interneurons and in pyramidal neurons. Our finding that endogenous calcium buffers had a higher level in interneurons may primarily explain the different kinetics and amplitudes of calcium transients. Synaptic stimulation-evoked calcium transients were also larger at distant dendritic locations. The spread of these signals was restricted to 12-13 $\mu \mathrm{m}$ long dendritic compartments. Supporting the reported lack of long-term potentiation in these interneurons, we found only sublinear or linear summations of calcium responses to coincident synaptic inputs and backpropagating spikes.
\end{abstract}

Key words: action potential; calcium [Ca]; hippocampus; imaging; interneuron; synaptic; dendrites; backpropagating two-photon imaging; calcium compartments

\section{Introduction}

Interplay between current synaptic activity and traces of previous activities may occur by the nonlinear summation of backpropagating action potentials (bAPs) and synaptic responses (Stuart and Sakmann, 1994; Stuart et al., 1997; Häusser et al., 2000). Active conductances in dendrites further increase the computational power of some neural systems (Yuste and Tank, 1996; Golding et al., 2002). The major sources of the calcium rise evoked by invasions of action potentials are the dendritic voltagesensitive calcium channels (VSCCs) in pyramidal neurons (Yuste and Denk, 1995; Schiller et al., 1998). VSCCs have a variable density and function along the dendrites of CA1 pyramidal neurons (Isomura et al., 2002). In hippocampal pyramidal neurons, coincident bAPs and synaptic stimulations can induce spine calcium rises larger than the linear combination of the individual signals (Yuste and Denk, 1995; Koester and Sakmann, 1998; Schiller et al., 1998). NMDA receptors may account for a large part of the supralinearity of the calcium increase during coinci-

\footnotetext{
Received Aug. 22, 2003; revised 0ct. 30, 2003; accepted 0ct. 30, 2003.

This study was supported in part by the Hungarian Research Fund (Grants OTKA T 034622, T 034622, and Ts 040736), Philip Morris, and the Hungarian Medical Research Foundation. We thank Dr. Karel Svoboda for his helpfu advice.

Correspondence should be addressed to E. Sylvester Vizi, Institute of Experimental Medicine, H-1083 Budapest, Szigony u. 43, Hungary. E-mail: esvizi@koki.hu.

DOI:10.1523/JNEUROSCI.3906-03.2004

Copyright $\odot 2004$ Society for Neuroscience $\quad$ 0270-6474/04/240661-10\$15.00/0
}

dent stimulations (Schiller et al., 1998). Although supralinear interactions between bAPs and synaptic inputs appear to support the current models of long-term potentiation (LTP) (Magee and Johnston, 1997; Stuart and Häusser, 2001), sublinear summation probably underlies long-term depression (LTD) (Linden, 1999).

Hippocampal CA1 and neocortical pyramidal neurons show a distance-dependent reduction in the amplitude of subsequent bAPs and in the evoked calcium transients in dendrites (Stuart and Sakmann, 1994; Spruston et al., 1995; Helmchen et al., 1999; Golding et al., 2002). Although, in the neocortical layer, $2 / 3$ pyramidal neurons bAP-evoked calcium transients are undetectable at a $140-150 \mu \mathrm{m}$ distance from the soma (Svoboda et al., 1999), in CA1 pyramidal neurons, the distance-dependent decline of calcium signals is relatively smaller at similar distances (Spruston et al., 1995). Attenuation of back-reporting signals stands parallel with the opposite scaling of synaptic potentials (Stuart et al., 1997). Various types of interneurons in the cerebral cortex also show the decremental scaling of bAP-evoked calcium signals up to 150-200 $\mu \mathrm{m}$ from the soma (Kaiser et al., 2001; Goldberg et al., 2003a). Dendritic bAPs are subject to modulation by various factors. Decrement of bAP-evoked dendritic calcium signals can be modified by cholinergic and GABAergic receptor activation (Tsubokawa and Ross, 1996, 1997; Xiong and Chen, 2002).

GABA-containing interneurons of the hippocampus provide crucial inhibition of projection cells to control their excitability, which leads to the synchronization of network activity (Freund 
and Buzsaky, 1996). Dendrites of CA1 interneurons are variably covered by excitatory and inhibitory synapses (Gulyas et al., 1999). However, scaling of synaptic activity and synaptic interactions with bAPs in dendrites of hippocampal interneurons are much less known. In the present work, we studied bAP-induced and synaptic stimulation-induced calcium signals in CA1 stratum radiatum interneurons. The main goal of this work was to understand the rules of local calcium mobilization by synaptic inputs and bAPs in different parts of the dendritic tree that shape neural integration in dendrites of CA1 stratum radiatum interneurons.

\section{Materials and Methods}

Slice preparation. Transverse slices $(300 \mu \mathrm{m})$ containing the hippocampus from 16- to 19-d-old Wistar rats were dissected as described previously (Zelles et al., 2001). Brain slices were placed in artificial CSF (ACSF) containing (in mM): $127 \mathrm{NaCl}, 25 \mathrm{NaHCO}_{3}, 25$ D-glucose, $2.5 \mathrm{KCl}, 2$ $\mathrm{CaCl}_{2}, 1 \mathrm{MgCl}_{2}$, and $1.25 \mathrm{NaH}_{2} \mathrm{PO}_{4}$, and incubated for $30 \mathrm{~min}$ at $34^{\circ} \mathrm{C}$. Slices were left at room temperature for $\geq 45 \mathrm{~min}$ before use.

Electrophysiology. Interneurons in the stratum radiatum of the CA1 hippocampal subfield were visualized under video infrared-differential interference contrast. Patch pipettes were pulled from borosilicate glass (1.2 mm OD; Harvard Instruments, March-Hugstetten, Germany). For current-clamp recordings, 5-9 $\mathrm{M} \Omega$ electrodes were filled with $125 \mathrm{~mm}$ K-gluconate, $20 \mathrm{~mm} \mathrm{KCl,} 10 \mathrm{~mm}$ HEPES, $10 \mathrm{~mm}$ Di-Tris-salt phosphocreatine, $0.3 \mathrm{~mm} \mathrm{Na}-\mathrm{GTP}, 4 \mathrm{~mm} \mathrm{Mg-ATP,} 10 \mathrm{~mm} \mathrm{NaCl}$, and 112/37 $\mu \mathrm{M}$ Oregon Green BAPTA-1. Cells with an initial resting membrane potential that was more negative than $-50 \mathrm{mV}$ were accepted. In most cases, five backpropagating action potentials were evoked by somatic current injection (90-170 pA; $15 \mathrm{msec} ; 5 \mathrm{APs} ; 29 \mathrm{~Hz}$ ) to induce dendritic calcium responses, because this provided a highly reproducible peak and a sufficient signal-to-noise ratio. Spike half-width did not change significantly during the experiment. Synaptic stimulation was performed with a unipolar glass microelectrode filled with ACSF. The electrode was positioned at $10-20 \mu \mathrm{m}$ from the dendrite, and the injected current $(6-20$ $\mu \mathrm{A} ; 0.1$ msec duration) was delivered by a stimulator (Supertech, Budapest, Hungary). Stimulus intensity was always maintained at a minimum to reach the threshold of synaptic responses. In each cell, we made $12 \pm$ 1.7 (mean \pm SEM) trials in average to confirm the presence of successes and failures. Data acquisition and analysis were performed using pClamp8 (Axon Instruments, Foster City, CA).

Two-photon imaging. Imaging was performed using a custom-made, two-photon, laser-scanning system consisting of a modified confocal microscope (Fluoview; Olympus, Hamburg, Germany) following the design of Karel Svoboda (Nimchinsky et al., 2001) and a titanium-sapphire laser (Millenia/Tsunami; SpectraPhysics, Fremont, CA) providing 100 fsec pulses at $80 \mathrm{MHz}$ at a wavelength of $820-840 \mathrm{~nm}$. Detection was performed in both epifluorescence and transfluorescence mode using external photomultiplier tubes (R3896; Hamamatsu, Herrsching, Germany). Colored glass filters (BG39; CVI Technical Optics) and dichroic mirrors (750DCSX, 750DCXXR; Chroma Technology, Brattleboro, VT) were used to separate emitted fluorescence from excitation light. To minimize photodamage, the intensity of the excitation laser light was always maintained at the minimum required to attain sufficient signalto-noise ratio. This intensity enabled us to perform continuous line scans over a dendrite for $10 \mathrm{~min}$ without photodamage or photobleaching, which corresponds the total recording time during an experiment. Hightime resolution fluorescence measurements were obtained in line-scan mode $(6,144 \mathrm{msec}$ temporal resolution) after zooming onto a dendritic section. Data recording started 20-25 min after break-in. During the experiments, the amplitude of bAP-evoked calcium signals decreased with time because of the continuous loading. Our observations are in agreement with those of Sabatini et al. (2002). To avoid errors in the estimation of the amplitude measured at different time points, we performed line-scan recordings at different locations randomly in time. The ratio of amplitudes of bAP-evoked calcium transients at two different locations remained constant in time, despite the time-dependent decline in the amplitude that was observed in both points $(n=2)$. Therefore, we repeatedly recorded bAP-evoked calcium responses at a particular distance (usually $40-50 \mu \mathrm{m}$ from the soma) and used it as a reference point for time-lapse amplitude normalization. In this way, the effect of time was mostly eliminated from the estimation of transient amplitudes during the course of the experiments. At the end of each experiment, a series of images across the depth of the volume encompassing the imaged neuron was taken. Image data were analyzed off-line using a custom-made program written in Matlab. Fluorescence traces are expressed as relative fluorescence changes $\left[\Delta F / F=\left(F-F_{0}\right) / F_{0}\right]$, where $F_{0}$ is the backgroundcorrected prestimulus fluorescence. Amplitude of the calcium transient was determined by the averaging of 30 data points at approximately the largest $\Delta F / F$ value of the transient. In a set of experiments, changes in fluorescence were converted to calcium concentrations. For transient change in the concentration of the calcium, the following equation (Maravall et al., 2000) is used:

$$
\frac{\Delta \mathrm{Ca}}{K_{D}{ }^{(F)}}=\frac{f_{\max }}{f_{0}}\left(1-R_{f}^{-1}\right) \frac{\delta f}{\left(\delta f_{\max }-\delta f\right) \delta f_{\max }},
$$

where $f_{0}$ is the fluorescence intensity at rest, $f_{\max }$ is the intensity when Oregon Green BAPTA-1 is completely bound to calcium, $K_{D}{ }^{(F)}$ is the dissociation constant (206 nM) (Maravall et al., 2000), $\delta f$ is the relative change in fluorescence, and $\delta f_{\max }$ is the maximum change in the fluorescence of the indicator after a saturating calcium influx. We measured $\delta f_{\max }$ values by inducing trains of action potentials ( $\left.56 \mathrm{~Hz} ; 20 \mathrm{APs}\right) . \delta f_{\max }$ was $1.6 \pm 0.2$ (mean $\pm \mathrm{SD}$ ), $R_{f}$ is the ratio of maximal and minimal fluorescence, and $R_{f}$ values were calculated in situ $\left(R_{f}, 3.68 \pm 0.89\right)$ and in cuvette $\left(R_{f}, 8.02 \pm 1.6\right)$. The resting calcium concentration can be calculated from the following:

$$
\frac{\left[\mathrm{Ca}^{2+}\right]_{0}}{K_{D}{ }^{(F)}}=\frac{\left(1-R_{f}^{-1}\right)}{\delta f_{\max }}-R_{f}^{-1},
$$

We estimated the calcium-binding capacity from the ratio of the change in a free calcium concentration $\left(\Delta\left[\mathrm{Ca}^{2+}\right]\right)$ and the change in a concentration of calcium bound to the fluorophore $(\Delta[\mathrm{FCa}])$ for pyramidal neurons and interneurons (Neher and Augustine, 1992):

$$
\kappa_{F}=\frac{\Delta[F \mathrm{Ca}]}{\Delta\left[\mathrm{Ca}^{2+}\right]}=\frac{K_{D}^{(F)}[F]_{\text {tot }}}{\left(K_{D}^{(F)}+\left[\mathrm{Ca}^{2+}\right]_{0}\right)\left(K_{D}^{(F)}+\left[\mathrm{Ca}^{2+}\right]_{\text {peak }}\right)},
$$

where $\left[\mathrm{Ca}^{2+}\right]_{0}$ and $\left[\mathrm{Ca}^{2+}\right]_{\text {peak }}$ are the calcium levels at rest and after the calcium inflow, and $[F]_{\text {tot }}$ is the total concentration of the fluorescent dye. We plotted $1 / \Delta \mathrm{Ca}^{2+}$ as a function of $\kappa_{F}$; and the endogenous calcium-binding capacity $\left(\kappa_{B}\right)$ was computed from the intersection of the fit with the $x$-axis.

Modeling calcium dynamics in dendrites of interneurons. Diffusion was modeled as a random walk process, with each calcium ion moving in the $x y z$ dimensions independently of other molecules. That is, during every time step $(\Delta t)$, an ion moves in either direction with the same probability. Consequently, it ends up in one of two locations with the probability, $P$, and remains at its initial site with the probability $1-2 \cdot P$. The same process also occurs in the $y$ or $z$ directions. After a large number of time steps, the probability of finding the molecule at any grid point in space will approach a Gaussian distribution centered at approximately the point of departure, and, overall, the calcium ion spreads in three dimensions with an effective diffusion coefficient, $D_{\mathrm{Ca}}$.

$$
D_{\mathrm{Ca}}=\frac{(\Delta x)^{2}}{4 \Delta t} .
$$

To assure that $D_{\mathrm{Ca}}$ has the value of 0.223 (Helmchen, 1999), $\Delta t$ was calculated directly from Equation 1. ( $\Delta t$ is in the order of $20 \mathrm{nsec}$ ). Steady-state level of buffers and calcium ions was calculated using the following equations:

$$
\begin{gathered}
{[B]_{\mathrm{tot}}=[B]+[B \mathrm{Ca}],} \\
{[F]_{\mathrm{tot}}=[F]+[F \mathrm{Fa}],} \\
{[\mathrm{Ca}]_{\mathrm{tot}}=[\mathrm{Ca}]+[B \mathrm{Ca}]+[\mathrm{FCa}],}
\end{gathered}
$$


where $[B]_{\text {tot }}$ is the total buffer concentration, $[\mathrm{BCa}]$ is the buffer-bound calcium, and $[F C a]$ is the fluorophore-bound calcium. $[F],[\mathrm{Ca}]$, and $[B]$ are the free concentrations for the fluorophore, calcium, and endogenous buffers. To model the temporal changes of intracellular calcium concentration, we used the following equations:

$$
\frac{\partial[\mathrm{XCa}]}{\partial t}=k_{\text {inX }}[\mathrm{Ca}][\mathrm{X}]-k_{\text {out } X}[\mathrm{XCa}],
$$

for $X=B$ or $X=F$. $k_{i n X}$ was $1 \times 10^{8} \mathrm{M}^{-1} / \mathrm{sec}$ for $X=B$ (Sabatini and Regehr, 1998) and 5 [times $10^{8} \mathrm{M}^{-1} / \mathrm{sec}$ for $X=F$ (Sabatini and Regehr, $1998), k_{\text {out } F}=K_{D}{ }^{(\mathrm{F})} \times k_{i n F}$ for $X=F$, and $k_{\text {out } B}=K_{D}{ }^{(B)} \times k_{i n B}$ for $X=$ $B . K_{D}{ }^{(B)}$ was set to $50 \mu \mathrm{M}$ (Sabatini and Regehr, 1998). Factors of calcium entry and removal were calculated as follows:

$$
\frac{\partial[\mathrm{Ca}]}{\partial t}=-\frac{\partial[\mathrm{BCa}]}{\partial t}-\frac{\partial[\mathrm{FCa}]}{\partial t}+I_{\mathrm{Ca}}^{N}-\gamma[\mathrm{Ca}]+I_{\text {leak }},
$$

where $I_{\mathrm{Ca}}{ }^{N}$ is the calcium current, $\gamma[\mathrm{Ca}]$ is the uptake of calcium, and $I_{\text {leak }}$ is the leak current into the intracellular space. For a single free molecule in the matrix, the probability $(P)$ of the binding of the calcium indicator or endogenous buffer molecule is given by the following:

$$
P_{b 1}=1-\exp \left(-k_{\mathrm{inX}} \times \delta t \times c_{1}\right),
$$

where $c_{1}$ is the concentration of one molecule in the matrix. For the unbinding of calcium from the buffer or the fluorophore, the probability becomes:

$$
P_{b 1}=1-\exp \left(-k_{\text {outX }} \times \delta t\right) .
$$

Increasing the number of molecules to $m$, it becomes equal to:

$$
P_{b m}=1-\exp \left(1-P_{b 1}\right)^{m} .
$$

Volume of the model dendrite was segregated into cells, and each cell contained three subcells for $\mathrm{Ca}, B \mathrm{Ca}$, and $F \mathrm{Ca}$. The number of molecules in these subcells was $N_{\mathrm{Ca}}, N_{B \mathrm{Ca}}$, and $N_{F \mathrm{Ca}}$. Calcium ions freely moved between cells. For movement from one subcell, transient probability for $X=B$ or $X=F$ was calculated as follows:

$$
P_{\text {out } X}=1-\exp \left(-k_{\text {out } X} \times \delta t \times N_{X C a}\right) .
$$

For movement into one subcell, transient probability was calculated as follows:

$$
P_{\text {inX }}=1-\exp \left[-k_{\text {inX }} \times \delta t \times c_{1} \times N_{\mathrm{Ca}} \times\left(N_{X_{\mathrm{tot}}}-N_{X \mathrm{Ca}}\right)\right] .
$$

where the calculated total number of buffers or fluorophores in one subcell was $N_{B \text { tot }}$ and $N_{F t o t}$. Removal of calcium ions was calculated as an uptake process only over the surface of the dendrite as:

$$
P_{\text {uptake }}=1-\exp \left(-\gamma \times \delta t \times c_{1} \times N_{\mathrm{Ca}}\right),
$$

where $\gamma$ is the constant for the uptake. The model of calcium dynamics was set in balance using a probability of leak calcium flow:

$$
P_{\text {Leak }}=1-\exp \left[-\lambda \times \delta t \times \frac{\left(2.5 \mathrm{mM}-[\mathrm{Ca}]_{\text {border }}\right)}{2.5 \mathrm{mM}}\right] .
$$

Before the simulation of calcium transients, the model reaches a dynamic steady state for $\mathrm{Ca}, \mathrm{BCa}$, and $F \mathrm{Ca}$. Calcium influx in response to the arrival of action potentials was modeled with the above probabilities in matrix cells located at the membrane of the dendrite for the mean open time of VSCCs. Parameters ( $\eta$ and $\gamma$ ) were set to describe the averaged traces obtained from pyramidal neurons. Leak current $(\lambda)$ was set to maintain a provided resting calcium concentration ( $40 \mathrm{~nm}$ for pyramidal neurons, $80 \mathrm{~nm}$ for interneurons). Endogenous calcium buffer concentration was assumed in the range of 1 to $5 \mathrm{~mm}$. On a longer time scale, we changed the value of $B_{\text {tot }}$ and selected the best fitting run to the experi-
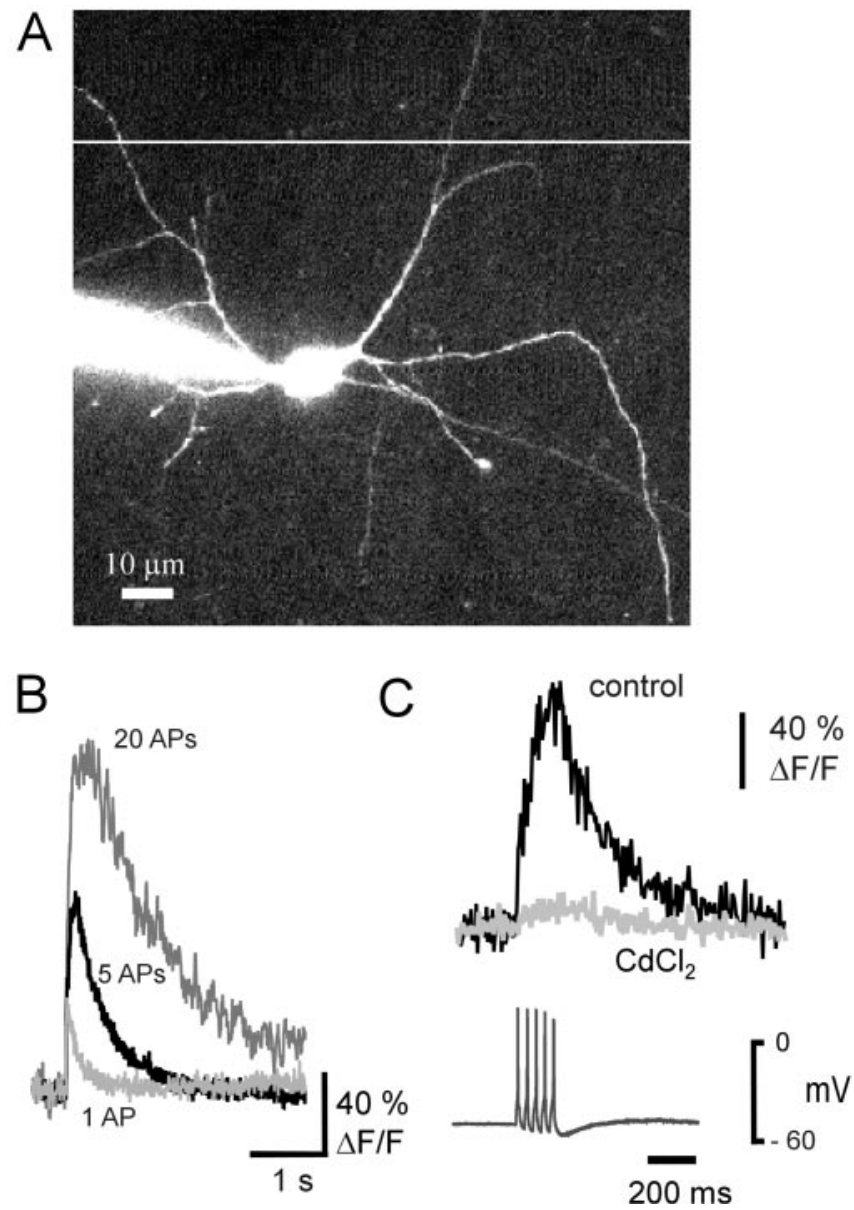

Figure 1. Imaging backpropagating spike-evoked calcium transients in hippocampal interneurons. $A$, Stratum radiatum interneurons in the CA1 region of the hippocampus were filled with Oregon Green BAPTA-1 (112 $\mu \mathrm{m})$ via a patch pipette. The position of the line scan on the dendrite is shown by a horizontal white line. $B$, Current injection into the soma induced 1-20 action potentials that evoked calcium transients in the dendrite. The signal in response to five action potentials $(29 \mathrm{~Hz}$ ) was not limited by the dye saturation, because 20 action potentials ( 56 $\mathrm{Hz}$ ) could cause a larger response. Five action potential responses provided an appropriate signal-to-noise ratio. Note that the increase in amplitude is not linear with the number of action potentials. $C, \mathrm{CdCl}_{2}$ given at $100 \mu \mathrm{m}$ concentration abolished the five action potential-evoked dendritic calcium response, indicating the primary role of voltage-sensitive calcium channels (sample traces).

mentally recorded transient that was used to estimate the calcium binding in interneurons in relation to pyramidal neurons.

\section{Results}

Backpropagating action potentials evoked calcium transients in dendrites of stratum radiatum interneurons

We performed combined electrophysiology and imaging experiments to characterize bAP-evoked calcium dynamics of dendrites of interneurons located in the stratum radiatum of the CA1 hippocampal region. Cell bodies were targeted with a patch electrode containing the high-affinity indicator Oregon Green BAPTA-1 at $112 \mu \mathrm{M}$ (Fig. 1A). We performed current injections into the somata of interneurons to induce action potentials that propagated to the dendrites and evoked calcium transients (Fig. $1 B$ ). Single action potentials caused calcium accumulations with small amplitude (Fig. $1 B$ ). Because of the higher signal-to-noise ratio for trains, we used five action potentials $(29 \mathrm{~Hz})$ to further study dendritic calcium dynamics. Trains seem to be more sensitive indicators of dendritic scaling of bAP-evoked calcium transients 
than single action potentials (Spruston et al., 1995; Svoboda et al., 1999). Longer trains at higher frequencies $(56 \mathrm{~Hz})$ caused substantially larger transients, suggesting that the five action potential-evoked calcium transients were not saturated in average (Fig. $1 B$ ). Cadmium chloride (100 $\mu \mathrm{M})$ caused a near-total block of the calcium rise without affecting the generation of action potentials. This finding suggests that VSCCs mediated most of the dendritic calcium influx in response to bAPs (Fig. 1C).

\section{Distance-dependent scaling of calcium transients evoked by backpropagating spikes}

Using the high-speed line-scan mode, we performed experiments to compare the relative amplitudes of bAP-evoked calcium transients along the dendrite (Fig. $2 A)$. To normalize the amplitude of the evoked transients during the time course of the experiment, we repeatedly measured transients in a reference point and in a distant location of the dendrite. The ratio of transient amplitudes was relatively stable, which subsequently helped us use the reference point (at 40-50 $\mu \mathrm{m}$ distance) for the time-lapse normalization of data (Fig. $2 B)$. Surprisingly, the amplitude of the dendritic calcium transient evoked by five bAPs $(29 \mathrm{~Hz})$ was incremental up to a $150-160 \mu \mathrm{m}$ distance from the soma $(n=$ 13 cells) (Fig. 2C,D). Single action potential-evoked calcium transients also exhibited incremental scaling; ratios of five AP- and single AP-evoked calcium transients did not show a significant tendency to increase or decrease with distance from the soma (data not shown). Evoking $500 \mathrm{msec}$ duration, $56 \mathrm{~Hz}$ trains of action potentials, the dendritic distribution of bAP-evoked calcium transients remained incremental ( $n=2$ cells; data not shown). The dendritic distribution of decay kinetics of bAP-evoked calcium transients showed very weak dependence on the distance from the soma independently on the dye concentration (Fig. 2E). As expected, the use of $37 \mu \mathrm{M}$ dye concentration revealed faster decay kinetics than the $112 \mu \mathrm{M}$ concentration. Nevertheless, the fast decay of calcium transients was found at virtually every distance, indicating that the possible fluctuation of calcium buffers alone did not determine the amplitude of these transients.

We analyzed the relationship between dendrite diameter and the peak of the bAP-evoked calcium transients. Responses were separated into a far group (70-120 $\mu \mathrm{m}$ from the soma) and a close group (10-60 $\mu \mathrm{m}$ from the soma). Close dendrites had a relatively wide range of diameters and transient amplitudes with a clear tendency for having smaller amplitudes in thicker dendrites (Fig. 2 F). Most of the far dendrites in our experiments had a diameter of $0.5-1 \mu \mathrm{m}$; however, the amplitudes of calcium transients still showed a rather homogenous distribution ranging from one of the smallest to the highest amplitude (Fig. $2 \mathrm{~F}$ ). These results revealed that dendritic calcium dynamics of these inter- neurons might be differently influenced by the diameter of the dendrite at different distances from the soma. Although close, most proximal sections exhibited a clear relationship between amplitude and diameter; more distant locations appeared to be independent on dendrite diameter. Overall, we can conclude that both diameter and distance from the soma in fact shape the distal increment in bAP-evoked calcium transients. To test to what extent the larger dendrite diameter causes a decrease in the amplitude of the local calcium transients, we performed Monte Carlo simulations of calcium dynamics assuming various dendrite diameters and constant VSCC density. This model confirmed the relationship between diameter and amplitude (Fig. $2 F$, gray line), which was similar to the experimentally observed relationship for close dendrites. These data suggest the primary role of the dendrite diameter in the distance-dependent scaling of bAP-evoked calcium transients in the more proximal parts of dendrites.

\section{Analysis of dendritic calcium transient waveforms}

To characterize the bAP-evoked dendritic calcium transients, we further analyzed the main determinants of the response waveform and performed comparisons to pyramidal neurons under 
A
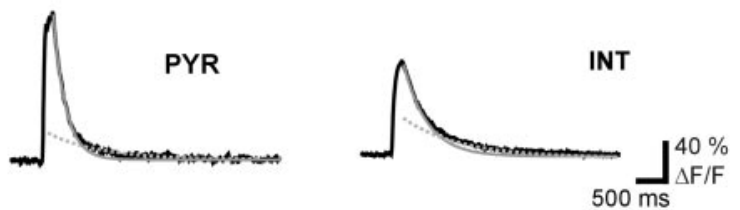

B
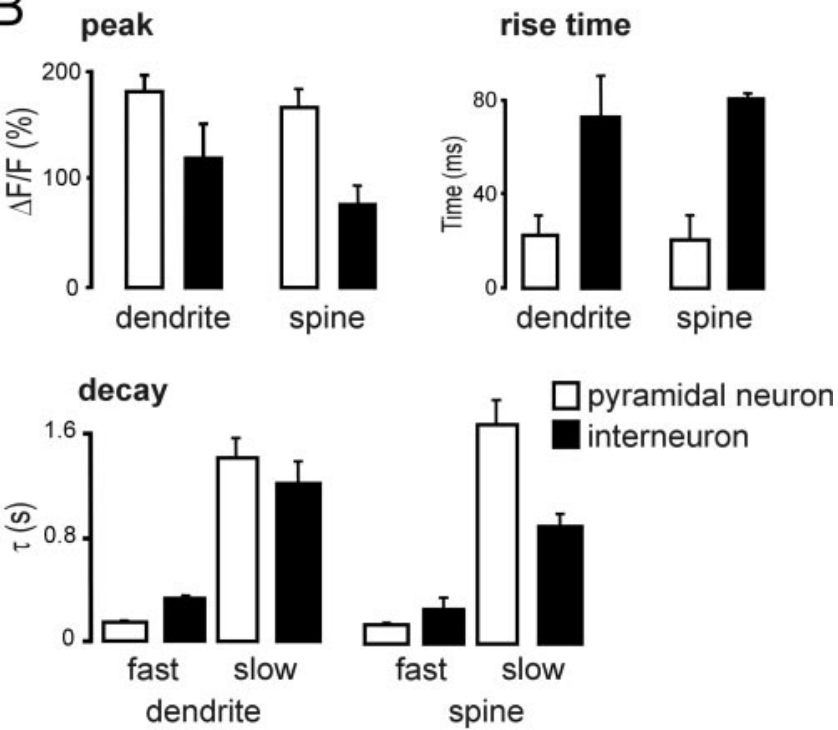

Figure 3. Analysis of calcium transient waveforms induced by backpropagating action potentials. A, Representative signal waveforms (black trace) and double decay fitting of action potential-evoked calcium transients in pyramidal neurons (PYR) and stratum radiatum interneurons (INT; fast component of the decay, solid line; slow component, dotted line) in response to five action potentials $(29 \mathrm{~Hz})$. Data presented here are averages of 25 sample traces from five slices. $B$, Peak amplitudes, decay time constants, and rise times for backpropagating spikeevoked calcium transients ( 5 action potentials at $29 \mathrm{~Hz}$ ) in pyramidal neurons and stratum radiatum interneurons. Data presented are means \pm SDs. Changes in decay ( $\tau$ values) are represented as fast and slow components. Error bars represent the error of the fit for the decay time constants ( $p<0.001$; unpaired $t$ test).

the same buffering conditions. Given the variability of calcium transients measured with different dyes or different concentrations of the dye (Kaiser et al., 2001; Sabatini et al., 2002), identical conditions for amplitude measurement of calcium transients are essential for the comparison of cell types. Despite the difficulty to specify "equivalent" dendritic sections between interneurons and pyramidal neurons, we sampled structures having similar geometrical constraints in the two cell types by focusing our measurements on $2 \mu \mathrm{m}$ diameter dendrites with a similar distance from the soma $(50-60 \mu \mathrm{m})$. For a complete comparison of the dendritic structure between cell types, we also measured transients in spines of both interneurons and pyramidal neurons in these experiments. We note that in spiny interneurons (75\%), only a few protrusions were observed. Overall, calcium transients in dendrites of interneurons were smaller and slower compared with similar signals recorded in pyramidal neurons (Fig. $3 A$ ). Average decay time constants and rise times of these responses in the two cell types were substantially different (Fig. $3 A, B$ ). In hippocampal pyramidal neurons, bAP-evoked calcium transients followed double exponential decay kinetics $(n=5)$. In interneuron dendrites and spines, intracellular bAP-evoked calcium transients also decayed with double exponential time constants containing a fast component and a slow component $(n=$ $9)$. The fast component of the decay was considerably shorter in pyramidal neurons, whereas the slow component was shorter in

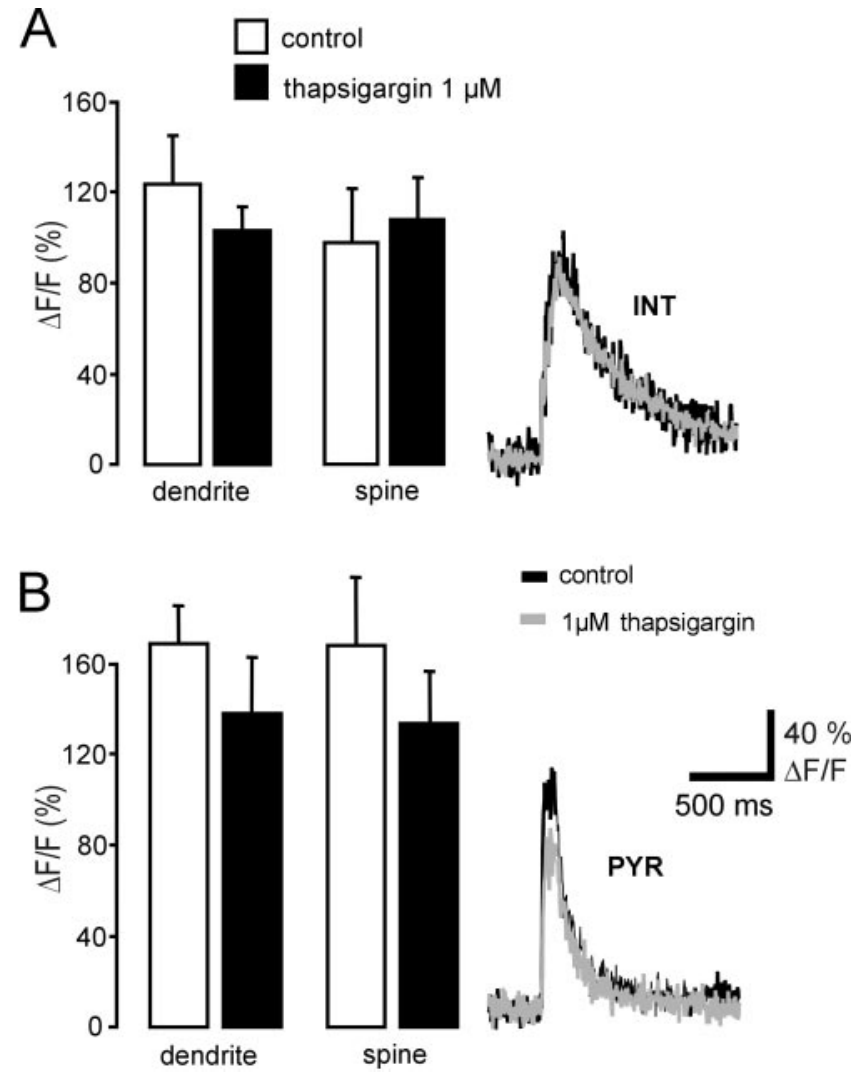

Figure 4. Effect of endogenous calcium store depletion. $A$, Treatment with $1 \mu \mathrm{m}$ thapsigargin failed to decrease the amplitude of bAP-evoked calcium transients ( 5 action potentials at 29 $\mathrm{Hz}$ ) measured at a distance of $50 \mu \mathrm{m}$ from the soma in spines and dendrites of interneurons (8 spines, 7 dendritic shafts, 5 cells). Data presented are means \pm SDs. Example traces for control and thapsigargin-treated conditions are shown at the right. $B$, In pyramidal neurons, the mean amplitude of bAP-evoked calcium transients ( 5 action potentials at $29 \mathrm{~Hz}$ ) was reduced both in dendritic shafts and spines after the store depletion ( $p<0.01 ; 11$ spines and shafts, 4 cells). Data presented are means \pm SDs. Example traces for control and thapsigargin-treated conditions are shown at the right.

the spines of interneurons (Fig. 3B). The peak of calcium transients was smaller in interneurons, which indicates that normalization to the peak for an interneuron and a pyramidal neuron pair would result in a substantially slower decay for interneurons. Rise time of calcium responses was shorter in pyramidal neurons (Fig. 3B). These data suggest that bAP-evoked calcium signaling in interneurons has a wider time window for interactions with other incoming signals in the dendrite than in pyramidal neurons. These characteristics of interneurons may influence their synaptic plasticity. We speculated as to whether the difference in decay kinetics in interneurons might be attributable to the variability of calcium buffering by endogenous calcium stores. To address the extent to which intracellular calcium stores contribute to bAP-induced calcium transients, we used thapsigargin to block calcium-ATPase and depleted the internal stores. After thapsigargin pretreatment ( $1 \mu \mathrm{M}$; perfusion started 12 min before the measurements), bAP-evoked calcium transients were recorded in spines and dendrites of interneurons $(n=5)$ and pyramidal neurons $(n=4)$. It was found that 12 min completely abolished a caffeine-induced elevation in the intracellular calcium level (data not shown). In interneurons, we found no significant interaction between control and thapsigargin-treated groups in decay kinetics, peak amplitude, or rise time of calcium transients in spines and dendrites (Fig. 4A). These data suggest 
A

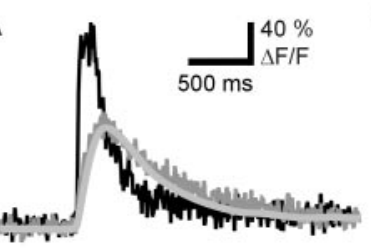

B

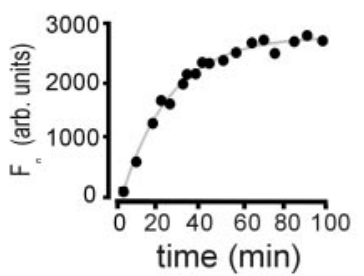

C

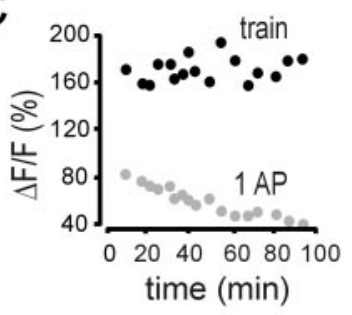

$\mathrm{D}$

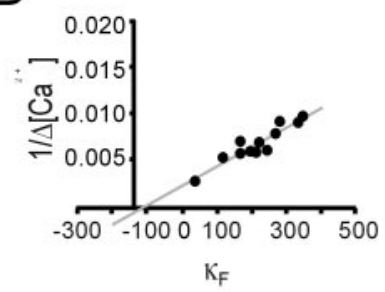

$E$

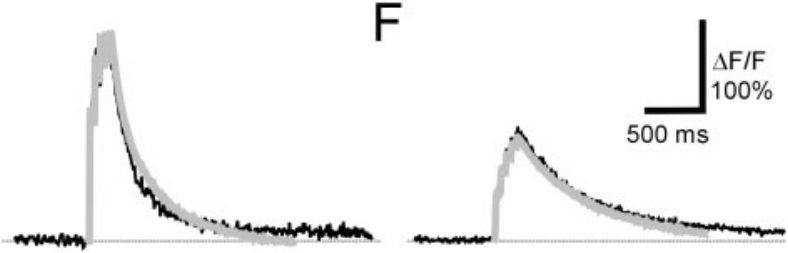

Figure 5. Calcium-binding capacity in pyramidal cells and interneurons shapes the decay of the transients. A, Backpropagating action potentials ( 5 action potentials at $29 \mathrm{~Hz}$ ) evoked calcium transients in pyramidal neurons (black trace). Using digital filtering, this transient could be converted to a trace (gray line) that showed high similarity with the calcium transient recorded experimentally in a stratum radiatum interneurons (gray trace). $B$, Time course of dye loading during an experiment. The baseline fluorescence $\left(F_{0}\right)$ was measured to estimate the amount of the loaded dye (filled circles). The optical plane was corrected for the occasional drift of the brain slice. Changes in $F_{0}$ fit to a single exponential (solid line). C, Amplitudes of calcium transients in response to trains of action potentials and a single action potential as a function of loading time. In a set of experiments, a larger train (16 action potentials at $56 \mathrm{~Hz}$ ) evoked dendritic calcium transients that were less sensitive to a time-dependent decrease in amplitude (filled circles). Amplitudes of single action potential-evoked signals declined as a function of time (open cir(les). D, Estimate of the endogenous buffer capacity. Endogenous calcium-binding capacity was computed from the $x$-axis intercept of the fit to inverse amplitude of bAP-evoked calcium transients. E, Monte Carlo simulation of dendritic calcium dynamics assuming a $1 \mathrm{~mm}$ endogenous buffer concentration resulted in a model trace (gray line) that appropriately described calcium transients evoked by backpropagating spikes ( 5 action potentials at $29 \mathrm{~Hz}$ ) in pyramidal neurons (black trace). $F$, For interneurons, the assumption of $4 \mathrm{~mm}$ endogenous buffer concentration provided the best fit (gray line) to a bAP-evoked calcium transient ( 5 action potentials at $29 \mathrm{~Hz}$; black trace) using Monte Carlo simulation.

that intracellular calcium stores do not contribute to bAP-evoked calcium transients in interneurons. In pyramidal neurons, the mean amplitude of the bAP-evoked calcium rise was reduced significantly $(p<0.01)$, by $20 \%$ in the spine and by $18 \%$ in the shaft, after the stores had been depleted (Fig. $4 B$ ). Decay kinetics remained double exponential for both spines and dendritic shafts in the presence of thapsigargin.

\section{Higher level of endogenous calcium buffers shapes the calcium transients in interneurons}

To explore the factors underlying the different response waveforms in pyramidal neurons and interneurons, we performed digital filtering of bAP-evoked signals recorded in a pyramidal neuron dendrite. Surprisingly, the filtered trace recorded from a pyramidal neuron provided a good fit to the transient recorded in an interneuron (Fig. 5A). Perhaps the most obvious filtering devices in the dendrite are the endogenous calcium buffers. In addition, hippocampal interneurons contain several classes of calcium-binding proteins that may substantially contribute to the clearance of calcium after a stimulation (Freund and Buzsaky, 1996). To determine whether the elongated bAP-evoked calcium response could be attributable to a larger endogenous buffer capacity in stratum radiatum interneurons, we estimated the endogenous calcium-binding ratio in dendritic shafts of interneurons and pyramidal neurons at a $40-50 \mu \mathrm{m}$ distance from the soma by recording bAP-evoked calcium transients during the loading of single cells with Oregon Green BAPTA-1 (112 $\mu \mathrm{M})$ as an exogenous calcium buffer (Neher and Augustine, 1992; Helmchen et al., 1996; Maravall et al., 2000). To ensure consistency across experiments, data of bAP-evoked transients were involved in the analysis if three criteria were met: (1) loading curve (amplitude vs time) was described with a single exponential fit; (2) data fell into a 40 min time window used for analysis; and (3) $(\Delta F / F)_{\max }$ values, determined by stimulations with trains, did not decline in reporting the vitality of cells (Fig. $5 B, C$ ). We also determined the resting intracellular calcium concentration, which was $37.1 \pm 9 \mathrm{~nm}$ ( 2 cells, 8 regions $)$ in pyramidal neurons, in close agreement with Maravall et al. (2000), whereas it was $81.4 \pm 28 \mathrm{~nm}$ ( 13 cells, 43 regions) in interneurons. The negative $x$-axis intercept provided the estimation of the endogenous calcium-binding ratio $\kappa_{B}$, because the calcium transient amplitude depends on the amount of the loaded indicator (Fig. 5D). We found the value of calcium-binding capacity $(\kappa)$ of $27 \pm 16$ ( 2 cells, 8 regions) in the case of CA1 pyramidal neurons. In interneurons, endogenous buffers were characterized by $\kappa=71 \pm 17$ (mean \pm SEM; 13 cells, 43 regions). Thus, the ratio of calciumbinding capacities of pyramidal neurons and interneurons was 1:2.6.

Next, on the basis of this cell-type difference between calciumbinding capacities, we established computer models to estimate the effect of this factor on the bAP-evoked calcium transients in pyramidal neurons and interneurons. We performed Monte Carlo simulations of dendritic calcium diffusion in the volume of dendrites. Assuming $2 \mu \mathrm{m}$ diameter dendrites, bAP-evoked calcium transients were compared in situations with different calcium binding. The model was calculated with the effect of buffering during the rising phase of the transient. In this way, different concentrations of calcium buffers (1-4 mM) provided various attenuation of calcium signals. Major parameters of the simulations included calcium diffusion, binding to the exogenous buffer, binding to endogenous buffers, and calcium extrusion. Dendritic calcium dynamics showed marked differences between interneurons and pyramidal neurons in the model and fit well with the recorded calcium transients (Fig. $5 E, F$ ). The best fitting traces for calcium transients of the pyramidal neuron and the interneuron were computed with the assumption of 1 and 4 $\mathrm{mm}$ of buffer, respectively. This 1:4 ratio of endogenous calcium buffer concentration between the two cell types provided a good approximation to the experimentally measured ratio (1:2.6). Therefore, this model favors the theory that the extra calciumbinding capacity in interneurons accounts for the elongated dendritic calcium transients in these cells.

\section{Distance-dependent scaling of synaptic stimulation-evoked calcium transients}

Backpropagating action potentials likely play an important role in integrating synaptic inputs by coincidence-dependent strengthening or weakening of synaptic potentials. In a set of experiments, we evoked synaptic calcium responses in dendritic shafts of interneurons by extracellular electrical stimulation. Synaptic activity evoked calcium transients with variable amplitudes 

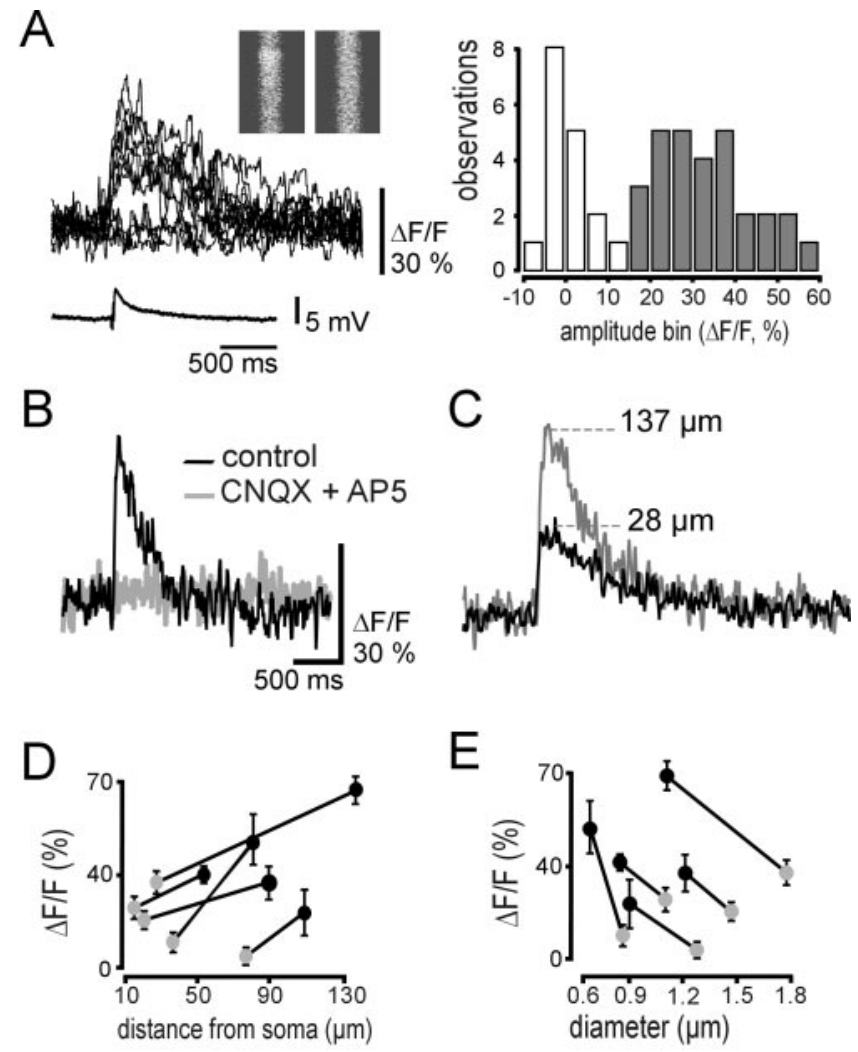

Figure 6. Distal enhancement of synaptic calcium responses. $A$, Synaptic stimulation induced excitatory postsynaptic potentials and calcium transients with variable amplitudes and failures in interneurons (sample traces; left). Inset, Line-scan images of examples of success and failure. Distribution of responses revealed two distinct groups of responses. Open bars represent frequency of failures, and the filled bars show the frequency of successes at different amplitude bins (right). $B$, When slices were perfused with AP-5 (40 $\mu \mathrm{m})$ and CNQX (10 $\mu \mathrm{m})$ to block AMPA and NMDA receptor-mediated excitatory synaptic transmission, no success was observed in response to synaptic stimulation (sample traces). C, Synaptic stimulation induced calcium transients with different amplitudes at proximal and distal dendritic sites (sample traces). D, Mean amplitudes of the synaptically evoked responses at the two dendritic locations as a function of their distance from the soma ( $n=5$ cells). Transients are larger at more distal dendrites (black circles) than in closer, proximal positions (gray circles). Error bars represent the within-site variability of trials (SEM). E, Dependence of amplitudes of synaptically induced calcium signals on the dendrite diameter. In these pairs ( $n=5$ cells), more distal dendrites (black circles) were always thinner than the more proximal site (gray circles), as reflected by the negative correlation between the amplitude and dendrite diameter.

and stochastic failures (Fig. 6A). Analysis of the amplitude distribution of synaptically evoked calcium transients revealed the two distinct populations of responses (Fig. 6A). During perfusion of the slices with blockers of excitatory synaptic transmission (CNQX, $10 \mu \mathrm{M}$; AP-5, $50 \mu \mathrm{M}$ ), only failures were observed after extracellular stimulation (Fig. $6 \mathrm{~B}$ ). Together, these data proved that calcium transients were evoked synaptically. To estimate distance dependence, we measured calcium dynamics at multiple sites of synaptic inputs along the dendrite. We located the stimulating electrode close to (at $\sim 15 \mu \mathrm{m}$ ) these dendritic sites to induce local synaptic transients using equal current intensities. This was important to avoid overlapping signals between the proximal and distal sites. We found that the mean amplitude of successes (as well as of all responses) increased at distal locations in all pairs $(n=5)$ (Fig. $6 C, D)$. Dendrite diameter was found to substantially influence the incremental scaling; at distal positions, the diameter was smaller in all cases than at proximal dendrites. This seems to be reflected by the amplitude of local calcium transients (Fig. 6E).
Compartments of synaptically evoked calcium transients in dendrites of interneurons

The fact that we could easily separate the synaptically evoked calcium transients along the dendrite initiated additional investigations on the spread of calcium signals in spine-free dendrites after extracellular stimulations. Perhaps the most appropriate experimental design of studying the extension of these signals is the scanning along a dendritic segment (Fig. 7Aa). In this way, we could observe simultaneous responses at subsequent locations in a segment after the synaptic stimulation. Occasionally, the extracellular stimulation produced calcium responses at two different input sites $6 \mu \mathrm{m}$ apart on a $15 \mu \mathrm{m}$ long dendrite segment (Fig. $7 A b$ ). These inputs were randomly active in time. Calcium transients in response to synaptic activity were the largest at the input sites, where the rise time of the transient was the shortest. Calcium signals spread over adjacent sections with decreasing amplitude (Fig. 7Ac). The wave-like spread was reflected by the increasing rise time of calcium transients measured at distant locations of the compartment (Fig. 7Ae). Assumption of the two individual compartments was supported by their different space constants and decay time constants (Fig. $7 A d$ ). To quantitatively characterize the size of the synaptic calcium compartments, we measured calcium transients with increasing distance from putative input sites using subsequent line scans that crossed the dendrite (Fig. $7 \mathrm{Ba}$ ). This method provided a sufficient number of data to estimate the mean length of the compartment (Fig. $7 \mathrm{Bb}$ ). In combining all data, the spreading of synaptic calcium transients was restricted to dendritic compartments of $\sim 6 \mu \mathrm{m}$ in length in one direction $(\sigma=5.9 \pm 0.3$; mean half width, 6.9; Gaussian fit) in spine-free dendrites of stratum radiatum interneurons (Fig. 7C).

\section{Summation of coincident bAPs and synaptic activity}

Finally, we tested the effect of the combination of the two stimulations (current injection to the soma and synaptic stimulation). Coincidence of postsynaptic depolarization and synaptic stimulation is thought to play an important role in synaptic plasticity. Stimulations (Fig. $8 \mathrm{~A}$ ) were applied within a $5 \mathrm{msec}$ time window. Dendrites appeared to separate into groups exhibiting a linear (Fig. $8 \mathrm{~B}$ ) or sublinear (Fig. $8 C$ ) summation of bAP-evoked and synaptic stimulation-evoked transients in stratum radiatum interneurons. The maximal difference of measured and calculated summations (Fig. 8D) was $-0.02 \pm 0.04$ for all cells (mean \pm SEM; $n=8$ ). Supralinear summation was not observed in dendritic shafts (Fig. 8D). Summation was independent, whether one or five action potentials were applied (data not shown). Values of $\Delta F / F_{\max }$ were also monitored in time to make sure that the saturation of fluorescent signals was avoided.

\section{Discussion}

Decremental distribution of bAP-induced calcium responses and distal boosting of synaptic events may represent an equalization effort in pyramidal neurons to avoid overamplification of incoming signals at distal sites during coincidence detection. In light of this assumption, the features of dendritic bAP-evoked and synaptically induced calcium transients in stratum radiatum interneurons are surprising and provide a distinct way to process dendritic signals. It is worth noting that, despite the large morphological and functional diversity of hippocampal interneurons (Freund and Buzsaky, 1996; Parra et al., 1998), the enhancement for both bAPs and synaptic activity in our experiments seemed consistent across cells. 
Incremental scaling of bAP-evoked calcium transients in CA1 stratum radiatum interneurons

Unlike cortical and hippocampal pyramidal neurons or cortical interneurons, bAPevoked dendritic calcium transients in CA1 stratum radiatum interneurons in this study showed a distance-dependent increment of up to $150-160 \mu \mathrm{m}$. Although, in hippocampal pyramidal neurons, an initial increment in calcium transients was found at a distance of 50-100 $\mu \mathrm{m}$ from the soma (Callaway and Ross, 1995), the evoked calcium signals are reported to decrease at a distance of $140-150$ $\mu \mathrm{m}$ (Spruston et al., 1995). The organization of the dendritic tree in cortical interneurons is very different from the pyramidal neurons; cortical layer $2 / 3$ bitufted interneurons also showed distancedependent attenuation of calcium responses after the backpropagation of one to four action potentials (Kaiser et al., 2001). In cortical fast spiking and irregular spiking interneurons, bAP-evoked calcium transients were also proximally restricted (Goldberg et al., 2003a). The possibility still exists that propagation of action potentials may improve with distance from the soma, and thus, as a consequence, larger calcium accumulation at distal sites occurs. It has been shown recently that the amplitude of bAPs does not decline while traveling in the dendrite of hippocampal oriens-alveus interneurons (Martina et al., 2000). However, even the assumption of nondecremental propagation of action potentials is insufficient to explain the larger bAP-evoked calcium transients in distal dendrites of stratum radiatum interneurons. It seems more likely that local amplification mechanisms of bAP-evoked events leads to the distally increased calcium response. Although proximal dendrites are more exposed to solution exchange with the recording pipette, washout of various soluble factors is not likely to explain the incremental relationship, because bAP-evoked calcium signals are decremental in pyramidal neurons and in other cell types using the same type of electrode for filling (Spruston et al., 1994; Kaiser et al., 2001; Goldberg et al., 2003a). Lower resting levels of intracellular calcium at distal locations may result in higher $\Delta F / F$ values. One possible candidate that may cause different resting calcium levels is certainly the endogenous calcium buffering. Endogenous buffers also influence the decay of calcium transients. Because of the linear correlation between $\mathrm{Ca}^{2+}$ pumping mechanisms and intracellular $\mathrm{Ca}^{2+}$ concentration, a higher $\mathrm{Ca}^{2+}$ level should associate with a faster decay (Tank et al., 1995) that partly explains the slight gradient in decay time constant with distance from the soma observed in our study (Fig. $2 E)$. Overall, we believe that the gradient in buffer concentration is not sufficient to fully explain the incremental scaling of bAPevoked transients. Uncaging of GABA over the dendrites of stratum radiatum interneurons revealed more functional GABA re-
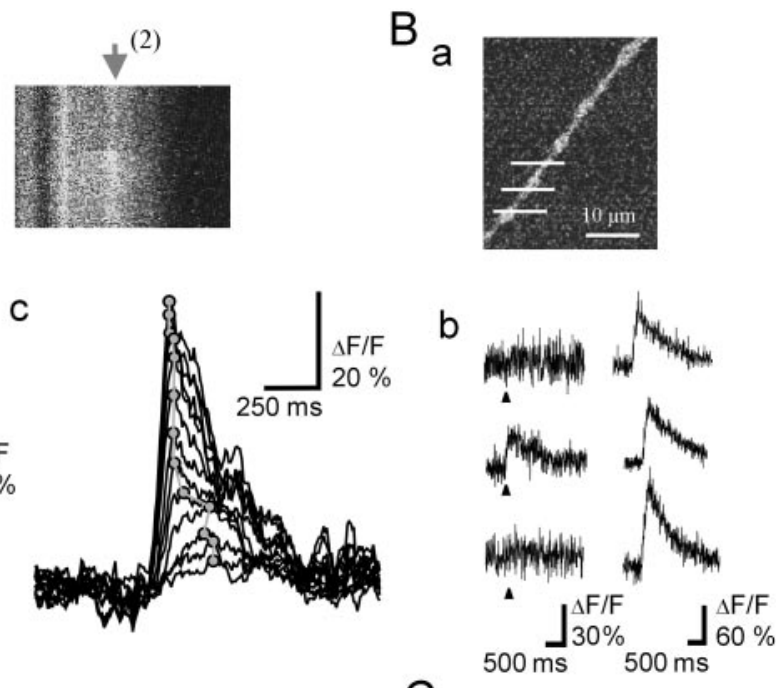

C
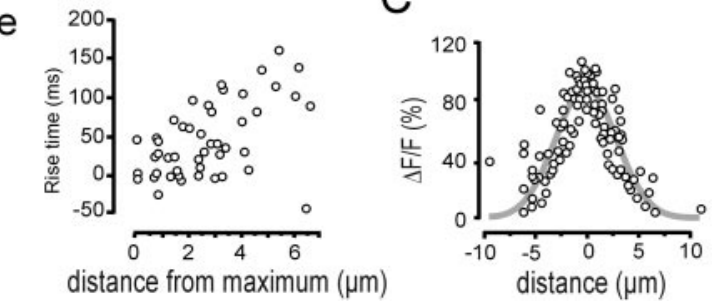

Figure 7. Synaptic calcium responses are organized into functional dendritic compartments. $A$, Scanning along the dendrite revealed two independent and compartmentalized synaptic inputs in a representative experiment. $a$, Representative line scan , whereas rise times become longer with distance (gray circles denote peaks). $d$, Mean peak amplitudes (amplitude, $\Delta F / F$ ) ( times of synaptically activated calcium responses within the compartment. $B$, Cross-scanning of dendrites revealed similar

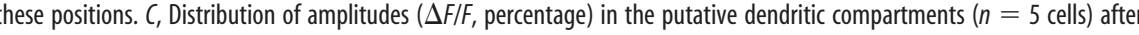
synaptic stimulation as a function of distance follows a Gaussian distribution and points to the site of the synaptic input. The largest synaptically induced $\Delta F / F$ values are plotted at different distances from the center of synaptic activity. Size of the synaptic response compartment is provided by the $\sigma$ of the Gaussian fit $(5.9 \pm 0.3 \mu \mathrm{m})$.

ceptors at proximal dendrites (Pettit and Augustine, 2000) that may indicate a decreasing inhibitory tone at distant dendrites. Therefore, the amount of calcium that a spike can mobilize may increase distally. In addition, distance-dependent distribution of potassium currents may also contribute to the scaling of bAPevoked calcium signals (Goldberg et al., 2003a).

\section{Dendritic scaling of synaptically induced calcium transients}

We reliably observed the distal enhancement of synaptic calcium responses in stratum radiatum interneurons at sites of up to 140 $\mu \mathrm{m}$ from the soma. The distance-dependent increase in the number of NMDA receptors in the proximal dendrites of somatostatine-positive interneurons (Nyiri et al., 2003) is in line with our observations that synaptically induced calcium responses are larger at distant locations. Supporting this, a distally directed gradient of glutamate receptors in stratum radiatum interneurons has been reported previously (Pettit and Augustine, 2000). The larger synaptic responses and occasional calcium spikes at distal dendritic sites of pyramidal neurons are likely 


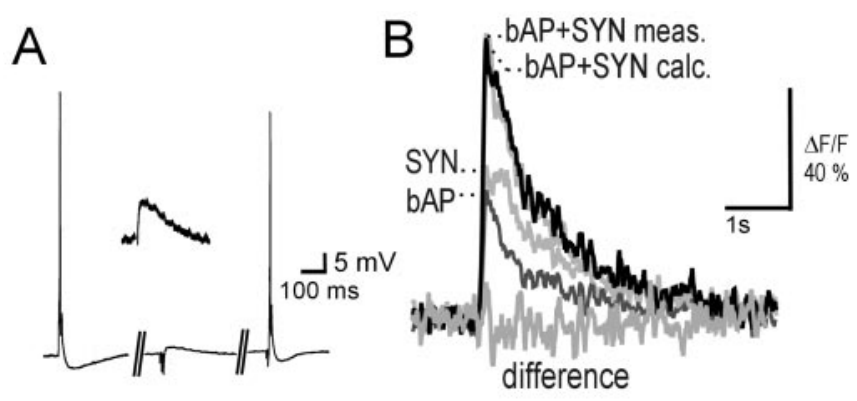

C
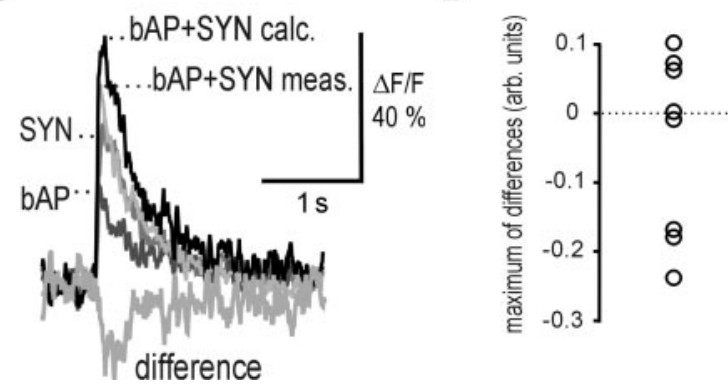

Figure 8. Summation of coincident signals in dendrites of hippocampal interneurons. $A$, Single backpropagating action potentials were induced by current injections to somata of CA1 stratum radiatum interneurons (left trace). Synaptic stimulation induced excitatory postsynaptic potentials (middle trace). Inset, Excitatory postsynaptic potential at a higher level of magnification. Synaptic stimulation and current injection were applied within a $5 \mathrm{msec}$ time window (right trace) to investigate the summation of these signals. $B$, Coincident synaptic activation (SYN) and bAP produced a larger calcium signal (bAP+SYN meas) in the dendrite (sample traces, $61 \mu \mathrm{m}$ from the soma). The difference of trace computed from the sum of the two individual stimuli (bAP + SYN calc) and bAP + SYN meas indicated a linear summation of calcium transients (linearity is shown by the difference) in this stratum radiatum interneuron. $C$, Sublinear summation of coincident activity (sample traces) in a dendrite of a different interneuron was also observed. The negative peak of the difference trace shows the sublinearity of the summation of presynaptic and postsynaptic stimuli. $D$, Summation of coincident stimuli seemed to form two groups, one including linearly summating sites (small peak difference values) and another including sites with sublinear summation (larger difference values).

essential to overcome the filtering effect of long dendrites (Stricker et al., 1996; Magee and Cook, 2000;Williams and Stuart, 2002). The range of soma distances in our experiments and the fact that calcium transients associated with spikes in the somatic recording were excluded from the analysis suggest that dendritic spikes did not interfere with the local calcium transients exhibiting distance dependence in our analysis.

\section{Cell-type differences in endogenous calcium buffers}

In layer $2 / 3$ of the neocortex, calcium-binding capacity was found lower in pyramidal neurons than in interneurons, whereas the bAP-evoked dendritic calcium transients had smaller amplitudes in interneurons (Kaiser et al., 2001). In our study, we went a step further in analyzing the effect of the larger calcium buffering in interneurons using Monte Carlo simulations. Although the digital filtering of the transient measured in pyramidal neurons was indicative of the possible role of endogenous calcium buffers, we found it insufficient for precise evaluation because of its lack of sensitivity to changes in rise time or geometrical constraints. Indeed, in pyramidal neurons, dendritic calcium dynamics depends on the surface/volume ratio in dendrites (Holthoff et al., 2002). Therefore, we performed Monte Carlo simulations of calcium signaling in dendrites with special regard to the volume in which calcium transients were generated. The resulting model provided calcium transients that could mostly explain the differences be- tween the recorded transients in pyramidal neurons and interneurons.

\section{Calcium compartments of synaptic activity}

We conclude that the approximate longitudinal size of the calcium compartment of single synaptic activity may extend over $\sim 12-13 \mu \mathrm{m}$ of the dendrite length (on the basis of the $\sigma$ value of the Gaussian fit). Despite the absence of spines in most dendritic sections, synaptic calcium responses seem to remain localized to compartments in neocortical interneuron dendrites (Goldberg et al., 2003a). The size of the dendritic compartments of cortical interneurons was $<15 \mu \mathrm{m}$ (Goldberg et al., 2003b). In our experimental conditions, a fraction of these synapses probably did not function because of the degradation of innervation in the slice preparation, which probably contributed to the sharper separation of compartments. In our study, compartments were characterized with calcium transients of different decays, suggesting a different amount of calcium buffering or calcium extrusion mechanisms within a $15-20 \mu \mathrm{m}$ dendritic section. The higher calcium buffer capacity in interneurons may help the separation of compartments. Although there are examples for interneuron dendrites sparsely covered by excitatory inputs (Gulyas et al., 1999), the separation of close synaptic inputs is not complete, because the calculated compartment length enables more than one synaptic contact even in sparsely innervated dendrites. Therefore, these compartments form a rather integrative unit for a group of synapses. Compartments of calcium responses to synaptic stimuli may associate to the overlapping domains of distinct AMPA receptor activation in interneuron dendrites (Walker et al., 2002; McBain, 2003). Direct stimulation did not likely cause a compartment-like distribution of responses of dendritic calcium dynamics, because (1) cases with no failures were excluded from that analysis, (2) cases for spontaneous synaptic activity showed similar responses in the dendrite, and, finally, (3) in a different set of experiments, CNQX plus AP-5 could block the activity using the same stimulus parameters at a similar distance from the dendrite.

\section{Summation of coincident activity in stratum radiatum interneurons}

Concurrent presynaptic and postsynaptic activation can induce LTP in synapses of pyramidal cells and can also produce supralinear summation in dendritic spines as a local and immediate mechanism contributing to LTP (Yuste and Tank, 1996). In stratum radiatum interneurons, a heterosynaptic form of LTD could be induced with distinct requirements for induction from pyramidal neurons (McMahon and Kauer, 1997; McBain et al., 1999). Here, we studied the summation of coincident bAP-evoked signals and synaptic responses in interneuron dendrites. Interestingly, in our study, dendritic shafts appeared to exhibit exclusively sublinear or linear summation during coincident synaptic activation and somatic action potential propagation. Sublinear responses can also detect coincident signals, increasing the computational power of the neuron. In a study by Koester and Sakmann (1998), sublinear summation of calcium responses was observed when action potentials preceded subthreshold synaptic potentials, whereas the reverse order stimulations resulted in supralinear responses. In contrast, in cortical interneurons, supralinear summation of coincident bAPs and synaptic activity has been found in dendritic sections adjacent to sites of synaptic inputs (Goldberg et al., 2003a). In agreement with our results, summation was linear at the synaptic site (Goldberg et al., 2003a).

In conclusion, this study revealed some of the properties of 
dendrites of inhibitory interneurons in the stratum radiatum. GABAergic inhibition represents an effective tool to synchronize the output of principal excitatory neurons of the hippocampus (Freund and Buzsaky, 1996). Therefore, dendritic integration in these interneurons has a major influence on the operation of the entire hippocampal neural network. Our finding that a distinct interplay between bAP-evoked and synaptic calcium responses exists in hippocampal interneurons supports the possibility of a separate regulation of the inhibitory and excitatory circuits by calcium mobilization within the hippocampus.

\section{References}

Callaway JC, Ross WN (1995) Frequency-dependent propagation of sodium action potentials in dendrites of hippocampal CA1 pyramidal neurons. J Neurophysiol 74:1395-1403.

Freund TF, Buzsaky G (1996) Interneurons of the hippocampus. Hippocampus 6:347-470.

Goldberg JH, Tamas G, Yuste R (2003a) $\mathrm{Ca}^{2+}$ imaging of mouse neocortical interneurone dendrites: Ia-type $\mathrm{K}^{+}$channels control action potential backpropagation. J Physiol (Lond) 551:49-65.

Goldberg JH, Yuste R, Tamas G (2003b) $\mathrm{Ca}^{2+}$ imaging of mouse neocortical interneurone dendrites: contribution of $\mathrm{Ca}^{2+}$-permeable AMPA and NMDA receptors to subthreshold $\mathrm{Ca}^{2+}$ dynamics. J Physiol (Lond) 551:67-78.

Golding NL, Staff NP, Spruston N (2002) Dendritic spikes as a mechanism for cooperative long term potentiation. Nature 418:326-330.

Gulyas AI, Megias M, Emri Z, Freund TF (1999) Total number and ratio of excitatory and inhibitory synapses converging onto single interneurons of different types in the CAl area of the rat hippocampus. J Neurosci 19:10082-10097.

Häusser M, Spruston N, Stuart G (2000) Diversity and dynamics of dendritic signaling. Science 290:739-744.

Helmchen F (1999) Dendrites as biochemical compartments. In: Dendrites (Stuart G, Spruston N, Häusser M, eds), pp 161-192. Oxford: Oxford UP.

Helmchen F, Imoto K, Sakmann B (1996) $\mathrm{Ca}^{2+}$ buffering and action potential-evoked $\mathrm{Ca}^{2+}$ signaling in dendrites of pyramidal neurons. Biophys J 70:1069-1081.

Helmchen F, Svoboda K, Denk W, Tank DW (1999) In vivo dendritic calcium dynamics in deep-layer cortical pyramidal neurons. Nat Neurosci 2:989-996.

Holthoff K, Tsay D, Yuste R (2002) Calcium dynamics of spines depend on their dendritic location. Neuron 33:425-437.

Isomura $\mathrm{Y}$, Fujiwara-Tsukamoto $\mathrm{Y}$, Imanishi $\mathrm{M}$, Nambu $\mathrm{A}$, Takada $\mathrm{M}$ (2002) Distance-dependent $\mathrm{Ni}^{2+}$-sensitivity of synaptic plasticity in apical dendrites of hippocampal CA1 pyramidal cells. J Neurophysiol 87:1169-1174.

Kaiser KMM, Zilberter Y, Sakmann B (2001) Back-propagating action potentials mediate calcium signaling in dendrites of bitufted interneurons in layer 2/3 of rat somatosensory cortex. J Physiol (Lond) 535:17-31.

Koester HJ, Sakmann B (1998) Calcium dynamics in single spines during coincident pre- and postsynaptic activity depend on relative timing of back-propagating action potentials and subthreshold excitatory postsynaptic potentials. Proc Natl Acad Sci USA 95:9596-9601.

Linden DJ (1999) The return of the spike: postsynaptic action potentials and the induction of LTP and LTD. Neuron 22:661-666.

Magee JC, Cook EP (2000) Somatic EPSP amplitude is independent of synapse location in hippocampal pyramidal neurons. Nat Neurosci 3:895-903.

Magee JC, Johnston D (1997) A synaptically controlled, associative signal for Hebbian plasticity in hippocampal neurons. Science 275:209-213.

Maravall M, Mainen ZF, Sabatini BL, Svoboda K (2000) Estimating intracellular calcium concentrations and buffering without wavelength ratioing. Biophys J 78:2655-2667.

Martina M, Vida I, Jonas P (2000) Distal initiation and propagation of action potentials in interneuron dendrites. Science 287:295-300.

McBain CJ (2003) Transient compartmentalization of interneuron dendrites. J Physiol (Lond) 555:1.
McBain CJ, Freund TF, Mody I (1999) Glutamatergic synapses onto hippocampal interneurons: precision timing without lasting plasticity. Trends Neurosci 22:228-235.

McMahon LL, Kauer JA (1997) Hippocampal interneurons express a novel form of synaptic plasticity. Neuron 18:295-305.

Neher E, Augustine GJ (1992) Calcium gradients and buffers in bovine chromaffine cells. J Physiol (Lond) 450:273-301.

Nimchinsky EA, Oberlander AM, Svoboda K (2001) Abnormal development of dendritic spines in FMR1 knock-out mice. J Neurosci 21:5139-5146.

Nyiri G, Stephenson FA, Freund TF, Somogyi P (2003) Large variability in synaptic $\mathrm{N}$-methyl-D-aspartate receptor density on interneurons and a comparison with pyramidal-cell spines in the rat hippocampus. Neuroscience 19:347-363.

Parra P, Gulyás AI, Miles R (1998) How many subtypes of inhibitory cells in the hippocampus? Neuron 20:983-993.

Pettit DL, Augustine GJ (2000) Distribution of functional glutamate and GABA receptors on hippocampal pyramidal cells and interneurons. J Neurophysiol 84:28-38.

Sabatini BL, Regehr W (1998) Optical measurement of presynaptic calcium currents. Biophys J 74:1549-1563.

Sabatini BL, Oertner TG, Svoboda K (2002) The life cycle of $\mathrm{Ca}^{2+}$ ions in dendritic spines. Neuron 33:439-452.

Schiller J, Schiller Y, Clapham DE (1998) NMDA receptors amplify calcium influx into dendritic spines during associative pre- and postsynaptic activation. Nat Neurosci 1:114-118.

Spruston N, Jaffe DB, Johnston D (1994) Dendritic attenuation of synaptic potentials and currents: the role of passive membrane properties. Trends Neurosci 17:161-166.

Spruston N, Schiller Y, Stuart G, Sakmann B (1995) Activity-dependent action potential invasion and calcium influx into hippocampal CA1 dendrites. Science 268:297-300.

Stricker C, Field AC, Redman SJ (1996) Statistical analysis of amplitude fluctuations in EPSCs evoked in rat CA1 pyramidal neurones in vitro. J Physiol (Lond) 490:419-441.

Stuart G, Sakmann B (1994) Active propagation of somatic action potentials into neocortical pyramidal cell dendrites. Nature 367:69-72.

Stuart G, Spruston N, Sakmann B, Häusser M (1997) Action potential initiation and backpropagation in neurons of the mammalian CNS. Trends Neurosci 20:125-131.

Stuart GJ, Häusser M (2001) Dendritic coincidence detection of EPSPs and action potentials. Nat Neurosci 4:63-71.

Svoboda K, Helmchen F, Denk W, Tank DW (1999) Spread of dendritic excitation in layer $2 / 3$ pyramidal neurons in rat barrel cortex in vivo. Nat Neurosci 2:65-73.

Tank DW, Regehr WG, Delaney KR (1995) A quantitative analysis of presynaptic calcium dynamics that contribute to short-term enhancement. J Neurosci 15:7940-7952.

Tsubokawa H, Ross WN (1996) IPSPs modulate spike backpropagation and associated $\left[\mathrm{Ca}^{2+}\right]_{\mathrm{i}}$ changes in the dendrites of hippocampal CA1 pyramidal neurons. J Neurophysiol 76:2896-2906.

Tsubokawa H, Ross WN (1997) Muscarinic modulation of spike backpropagating in the apical dendrites of hippocampal CA1 pyramidal neurons. J Neurosci 17:5782-5791.

Walker HC, Lawrence JJ, McBain CJ (2002) Activation of kinetically distinct synaptic conductances on inhibitory interneurons by electrotonically overlapping afferents. Neuron 35:161-171.

Williams SR, Stuart GJ (2002) Dependence of EPSP efficacy on synapse location in neocortical pyramidal neurons. Science 295:1907-1910.

Xiong W, Chen WR (2002) Dynamic gating of spike propagation in the mitral cell lateral dendrites. Neuron 34:115-126.

Yuste R, Denk W (1995) Dendritic spines as basic functional units of neuronal integration. Nature 375:682-684.

Yuste R, Tank DW (1996) Dendritic integration in mammalian neurons, a century after Cajal. Neuron 16:701-716.

Zelles T, Franklin L, Koncz I, Lendvai B, Zsilla G (2001) The nootropic drug vinpocetine inhibits veratridine-induced $\left[\mathrm{Ca}^{2+}\right]_{\mathrm{i}}$ increase in rat hippocampal CA1 pyramidal cells. Neurochem Res 26:1095-1100. 